\title{
O USO PROLONGADO DE BENZODIAZEPÍNICOS POR IDOSOS E O RISCO PARA DEMÊNCIA
}

THE BENZEDIAZEPINE FOR A LONG TIME IN ELDERLY PEOPLE AND THE RISK FOR DEMENTIA

LUCIANA SILVEIRA FARIA ${ }^{1}$; JOSIANE BUDNI2*

'Pós-Graduação em Saúde Mental; Universidade do Extremo Sul Catarinense, UNESC, Criciúma, SC, Brasil.

'Laboratório de Doenças Neurodegenerativas; Laboratório de Neurociências; Programa de Pós-Graduação em Ciências da Saúde; Unidade Acadêmica de Ciências da Saúde -Universidade do Extremo Sul Catarinense - UNESC, Criciúma, SC, Brasil.

\section{Endereço para correspondência:}

* Autor correspondente: Laboratório de Neurociências, Programa de Pós-Graduação em Ciências da Saúde, Unidade Acadêmica de Ciências da Saúde, Universidade do Extremo Sul Catarinense, 88806-000 Criciúma, SC, Brazil;

E-mail: josiane.budni@unesc.net

Tel.: +55 (48) 3431-2792 


\section{RESUMO}

A população está envelhecendo e com isso, políticas de saúde precisam ser desenvolvidas, para fornecer maior qualidade de vida à população idosa. Há um grande número de indivíduos idosos que administram vários medicamentos em função das comorbidades do envelhecimento. Entre estes fármacos estão os benzodiazepínicos. Estes causam vários efeitos colaterais, entre eles a amnésia, uma queixa constante dos idosos. Portanto, estudo tem por objetivo relacionar o uso prolongado de benzodiazepínicos em idosos e o risco para o desenvolvimento da demência. Alguns estudos mostram que vários sintomas que antecedem a demência são tratados com benzodiazepínicos. São vários os fatores de risco atribuídos ao uso desses fármacos em idosos, como déficit cognitivo, quedas, fraturas, etc. A indicação do uso e seu tempo de uso, devem ser constantemente avaliados, pois seu uso prolongado causará muitos prejuízos a curto prazo. Estudos apontam um risco aumento para os indivíduos idosos em desenvolver demências com o uso prolongado de benzodiazepínicos. A população mundial está envelhecendo, e portanto, precisa-se do desenvolvimento de políticas públicas de saúde para as pessoas com mais de 60 anos para que estas sejam assistidas e que, inclusive, se possa ter maiores informações acerca do efeito prolongado de certos medicamentos, como os benzodiazepínicos. Adicionais pesquisas relacionadas aos fármacos que possam ser utilizados por idosos e que não comprometam a saúde dos mesmos, e sim aumentam a qualidade de vida destes, são necessários.

Palavras-chave: benzodiaepinico; idoso; demência.

\section{ABSTRACT}

The population is aging and with this, health policies need to be developed, to provide a higher quality of life for the elderly population. There are a large number of elderly individuals who administer various medications depending on the comorbidities of aging. Among these drugs are benzodiazepines. These cause several side effects, among them amnesia, a constant complaint of the elderly. Therefore, a study aims to relate the prolonged use of benzodiazepines in the elderly and the risk for the development of dementia. Some studies have shown that several symptoms that precede dementia are treated with benzodiazepines. There are 
several risk factors attributed to the use of these drugs in the elderly, such as cognitive deficits, falls, fractures, etc. The indication of the use and its time of use, must be constantly evaluated, since its prolonged use will cause many damages in the short term. Studies point to an increased risk for elderly individuals in developing dementias with prolonged use of benzodiazepines. The world population is aging and therefore the development of public health policies is needed for people over the age of 60 to be assisted and even more information about the prolonged effect of certain drugs, Such as benzodiazepines. Additional research related to the drugs that can be used by the elderly and that do not compromise the health of the same, but increase the quality of life of these, are necessary.

Descriptors: benzodiaepinic; old man; insanity.

\section{INTRODUÇÃO}

O envelhecimento das pessoas em todo o mundo é um grande triunfo, mas também um desafio enorme para a humanidade. É muito difícil envelhecer em um pais em desenvolvimento, onde muitos idosos pertencem a uma estrutura familiar onde ainda são os principais provedores, estando sujeitos a uma pior qualidade de vida, ou então são os cuidadores de outros idosos, situação bem comum em todas as realidades. Nos próximos anos esse envelhecimento causará muitas demandas, principalmente econômicas e sociais, porém a população idosa é geralmente ignorada (GARRIDO E MENNEZES, 2002; WHO, 2005). Segundo a Organização Mundial de Saúde (OMS), os países podem custear o envelhecimento se implementarem programas e políticas. Hoje acredita-se que medidas para ajudar as pessoas mais velhas a se manterem ativas e hígidas é essencial. No mundo o número de pessoas com mais de 60 anos, cresce rapidamente mais que qualquer outra faixa etária. Entre 1970 e 2025 o crescimento esperado é de $223 \%$, em torno de 694 milhões de idosos. Em 2025 haverá 1,2 bilhões de pessoas com mais de 60 anos. Até 2050 haverá dois bilhões sendo desse número $80 \%$ em países em desenvolvimento. $\mathrm{O}$ aumento da longevidade e a redução das taxas de natalidade vão assegurar o contínuo agrisalhamento da população mundial. Nos países desenvolvidos, a população mais velha está envelhecendo também, o número de 
pessoas com mais de 80 anos chega a 69 milhões, a maior parte vive em regiões mais desenvolvidas. $O$ rápido envelhecimento nos países em desenvolvimento vem acompanhado de drásticas mudanças nas estruturas familiares. São necessários mais investimentos em pesquisas e programas que beneficiem os idosos, para poder assim alcançar um envelhecimento saudável (WHO, 2005; ONU, 2013; ONU, 2015).

O fundamental no envelhecimento é a autonomia com o intuito de poder continuar fazendo suas próprias escolhas e tomar suas decisões, cuidar de si mesmo, até quando possuam doenças crônicas. Entres estas incluem as doenças cardiovasculares, neoplasias malignas, doenças respiratórias crônicas, muscoesqueléticas, transtornos neurológicos e mentais (ISMAIL et al., 2011; TAZKARJ et al., 2016). O envelhecimento da população a nível mundial e o aumento com os gastos públicos com saúde e assistência social, são vistos como uma ameaça a estabilidade econômica. Uma intervenção efetiva para os idosos é muito complexa, por não haver o acesso aos cuidados de saúde, renda adequada, proteção social, todo tipo de ação deve ser holística, coordenada e centrada no idoso (PINCUS, 2017). Em 2002 aconteceu a Assembleia Mundial das Nações Unidas que focou na intenção de identificar as características de uma nova linguagem e abordagem ideológica quanto ao envelhecimento. É essencial a adoção de uma nova visão que garantiria uma participação continuada em questões sociais, econômicas, espirituais, culturais e cívicas e não a vida profissional longa e a atividade física. Essa política de saúde começou a ser preparada em 1999, quando a OMS celebrou o Dia Internacional do Idoso, onde aconteceu uma mobilização global que percorreu o mundo, que foi o Envelhecimento Ativo, que continuará existindo tanto como conceito quanto ferramenta. A OMS passou a defender a meta do Envelhecimento Ativo definido como processo de otimização das oportunidades de saúde, participação e segurança, com o objetivo de melhorar a qualidade de vida à medida que as pessoas ficam mais velhas. Nosso mundo está envelhecendo rapidamente, o legado do século XX é a longevidade. Nos dias de hoje o crescimento da população se deve a um menor número de pessoas morrendo a cada ano, do que o número de pessoas nascendo. Há mais mulheres idosas do que homens idosos, no padrão mundial as mulheres vivem 4,5 anos mais que os 
homens. Uma vida mais longa e saudável e as mudanças dos modelos de curso de vida em muitos países, fazem com que a imagem e o papel do idoso sejam redefinidas. Envelhecer é cada vez mais visto como um processo individual, com o prolongamento da jovialidade e com múltiplas oportunidades de desenvolvimento pessoa. $O$ envelhecimento ativo pode ser definido como resiliência, pode acessar as reservas necessárias para se adaptar, suportar e aprender com os desafios que vão surgindo ao longo da vida e que a saúde, segurança financeira, conhecimentos e habilidades representam as reservas para conseguir o crescimento pessoal, que levam ao bem-estar e à qualidade de vida (WHO, 2005).

Em todas as regiões do mundo o número de idosos com demência aumentará rapidamente, a não ser que medidas significativas de prevenção aumentem velozmente. A demência caracteriza-se por várias incapacidades, os sintomas comportamentais e psicológicos afetam diretamente a qualidade de vida tanto do idoso portador da doença, quanto da família, sendo causas bastante comuns para institucionalização. Nos próximos vinte anos está previsto um aumento acentuado em países de baixa e média renda de portadores de demência. Os números mostram que $58 \%$ das pessoas com demência vivem em países de baixa renda e que esse valor aumentara para $71 \%$ em 2050. O curso da doença não pode ser alterado, mas o diagnóstico precoce permitirá que os idosos consigam um planejamento de cuidados avançados enquanto ainda conseguem terem um entendimento. A educação, a formação e o apoio aos cuidadores são eficazes na redução da tensão do cuidador e na sua morbidade psicológica. Em relação aos países de alta renda retardar ou evitar a transição para as casas de saúde. Atualmente as pessoas com demência recebem o diagnostico tardiamente. A demência é uma das dez principais causas de mortalidade no mundo. Mais da metade das pessoas com demência no mundo vivem em países menos desenvolvidos. Os efeitos dessa doença progressiva são desgastantes nas famílias, que passam a apresentar maior risco de declínio da própria saúde física e emocional (ISMAIL et al., 2011; OTA et al., 2015; CUSTODIO et al., 2017; FRANÇOIS et al., 2017).

O mundo todo precisa erguer a bandeira do envelhecimento ativo, garantindo saúde, aprendizado, participação, segurança e proteção. A grande maioria dos 
países ricos, que tiveram o privilégio de enriquecer antes de envelhecer, tem programas e ambientes sociais, que de alguma forma vão suprir as necessidades dessa população, garantindo a sustentabilidade de todas as gerações (PINCUS et al., 2017). Países de renda média, com economias em expansão, estão envelhecendo como os países ricos, as melhorias estão sendo implantadas à passos lentos, devido à grande desigualdade social. Países de baixa renda, estão buscando o desenvolvimento econômico e social para a melhoria da vida de todos. Para conseguir extrair o máximo da longevidade é muito importante aumentar os anos com boa saúde e os anos de vida. Os fatores de risco em relação ao ambiente e de comportamento, precisam ser reduzidos, junto a eles os fatores de proteção devem ser intensificados para que as doenças crônicas e a parte funcional possam ser prevenidas ou adiadas. A promoção, prevenção e o diagnóstico correto das doenças, principalmente as demências, devem estar garantidas para poder existir 0 envelhecimento ativo. $\mathrm{O}$ cuidado deve ser integrado e personalizado enfatizando a manutenção da maior capacidade funcional e qualidade de vida possível. O envelhecimento ativo é um processo para a vida toda, quanto mais tempo se preparar com saúde e conhecimento maiores são as chances de desfrutar a velhice com muita qualidade de vida. A longevidade está modificando o curso de vida de várias formas. Homens e mulheres trabalham enquanto possuem capacidade física e mental compatíveis com a função que exercem sustentando suas famílias. A idade avançada costuma ser definida pela falta de capacidade e por depender de outras pessoas. A aposentadoria não se define mais pela falta de capacidade nos países mais desenvolvidos, é conhecida como uma fase privilegiada de satisfação e renovação pessoal. A capacidade de poder atuar em todas as atividades da vida depende de ter saúde conhecimento para a participação plena na sociedade. O envelhecimento levanta preocupações quanto a segurança econômica, gastos, oferta de mão de obra, receitas fiscais e crescimento da renda per capta. Mudanças de comportamento e políticas de saúde devem ser analisadas pensando no futuro das gerações. Devem acontecer transformações nas políticas públicas e privadas em relação a aposentadoria, outro fator é a ênfase na prevenção e detecção de doenças, ao invés de se pensar apenas no tratamento. Intervir na educação para ampliar a força de trabalho efetiva. Melhoras na saúde em prestar cuidados de longa 
duração. Quanto mais reformas públicas e institucionais acontecerem mais suaves serão os caminhos para ajuste da economia e aposentadoria a longo prazo, para melhoria da vida da população mais velha, se faz necessário um desenvolvimento global para uma sociedade mais justa e eficiente (GARRIDO E MENEZES, 2002; RAMOS, 2003; WHO, 2005; SANDERSON E SCHERBOV, 2015).

\section{Benzodiazepínicos}

O primeiro benzodiazepínico surgiu em 1959, o diazepam foi introduzido em 1963, desde então várias drogas derivadas dessas substancias foram introduzidas no mercado. Foram longamente prescritos para ansiedade e insônia nas décadas de 1960, 1970 e 1980. Os benzodiazepínicos são diferentes nas suas propriedades de absorção, distribuição e eliminação, mas semelhantes nas propriedades hipnóticaansiolítica, relaxante muscular e anticonvulsivante. Estão entre as drogas mais seguras, mas apresentam efeitos colaterais, sendo o mais comum a sonolência durante o dia, pode ocorrer amnésia, ansiedade, insônia, sintomas de abstinência e podem ainda prejudicar a psicomotricidade. Podem provocar amnesia anterógrada ocorrendo um déficit na consolidação das memorias. O uso crônico pode levar a comprometimentos cognitivos persistentes. Aos poucos foram aparecendo desconfianças em relação ao seu uso, no que diz respeito a dependência e o abuso. Surgiram problemas com a excessiva prescrição e com a falta de conhecimento e pratica de como é a sua ação e seus efeitos. Alguns profissionais de saúde não a prescrevem por falta de conhecimento e receio deixando de beneficiar alguns pacientes. Com o passar do tempo novos benzodiazepínicos surgiram, com diferenças em sua ação no organismo (Linden et al., 2004; Bernik, 2010).

Sabe-se muito sobre seus efeitos na potencialização da ação do GABA (ácido g-aminobutírico) que é o principal neurotransmissor inibitório do sistema nervoso central. A prática mostra que esses medicamentos devem ser utilizados por períodos curtos, levando em consideração aspectos clínicos e de personalidade na hora de prescrevê-los. Algumas pessoas necessitam de um uso mais prolongado, daí todo um cuidado envolvido por conta do risco da dependência e tolerância. Por isso é de fundamental importância o esclarecimento ao paciente de todos os riscos e benefícios na hora da escolha desse tipo de medicamento e tempo de tratamento. 
Os benzodiazepínicos são rapidamente absorvidos pelo organismo, possuem uma alta lipossolubilidade, conseguem atravessar a barreira hematoencefálica, também conseguem atravessar a barreira placentária aumentando os riscos de anomalias e má formações congênitas, passam para o leite materno sedando o recém-nascido. A retirada dos benzodiazepínicos deve ser lenta, pois pode promover síndromes de abstinência ou ansiedade de rebote. $O$ uso deve ser intermitente e breve, retirando a medicação assim que os sintomas forem desaparecendo. Essas drogas podem ser classificadas de acordo com a meia vida, tempo que a droga fica na corrente sanguínea, até que sua metade seja eliminada. Tem efeito de curta, média e longa duração. Os benzodiazepínicos de curta duração são os que apresentam maior risco de abuso (OLIVEIRA et al., 2011).

\section{Envelhecimento e Uso de benzodiazepínicos}

Os idosos podem sentir mais os efeitos dos benzodiazepínicos, no que diz respeito a memória, quando são acometidos de alguma doença que envolva a cognição. Por isso seus efeitos em relação a cognição têm sido cada vez mais estudados. Os efeitos sobre a memória variam muito, depende do tipo de memória avaliada podendo trazer prejuízos, ou até nem fazer diferença com o seu uso. Os efeitos crônicos causados pelos benzodiazepínicos, mostram que existe um prejuízo cognitivo e que este não é reversível após a parada da tomada da medicação. São usados por períodos maiores que os recomendados, que seriam de duas a quatro semanas. Seus efeitos em pacientes idosos comprometem todo o processo de absorção, distribuição, metabolismo e a excreção renal. Outros fatores importantes são: co-morbidades, polifarmácia, não adesão ao tratamento, uso abusivo, pois os idosos são muito mais sensíveis a toxicidade e apresentam uma sensibilidade aumentada aos efeitos colaterais no sistema nervoso central. A escolha do benzodiazepínico mais apropriado vai depender do perfil farmacológico de cada substância e do perfil clinico de cada paciente idoso. Todos sabem profissionais quanto usuários que são as substâncias mais difíceis de se interromper o uso, e que não se tem benefícios a longo prazo. Os benzodiazepínicos são os medicamentos mais usados em todo o mundo, esse consumo dobra a cada cinco anos (OLIVEIRA et al., 2011). 
Os benzodiazepínicos causam altas taxas de tolerância e dependência o que proporciona sempre ao aumento da dose, para que se obtenha o mesmo efeito terapêutico. Alguns estudos concluíram que o consumo por idosos com doses terapêuticas por um tempo maior que oito semanas, contribui para o desenvolvimento da dependência e do déficit cognitivo. A suspenção deve ser gradual e de forma lenta, é de extrema importância o esclarecimento ao paciente desde a escolha e conduta do tempo de tratamento, esclarecer e orientar sobre os riscos e cuidados a serem tomados, somente assim a retirada será bem-sucedida. Sua utilização em idosos deve ser criteriosamente analisada por conta de como se dá o processo de envelhecimento. Várias alterações metabólicas, como redução da excreção renal, do metabolismo hepático, perda da massa muscular associada ao aumento da gordura, geram uma mudança nos níveis plasmáticos e na meia vida dessas substâncias. Sendo o seu efeito potencializado causará vários riscos como sonolência diurna, quedas e déficit cognitivo. Os efeitos adversos são mais graves como depressão respiratória, apneia, parada respiratória e parada cardíaca. As reações paradoxais como inquietude, agressividade, alucinações, psicoses, comportamentos inapropriados, ilusão, raiva, são efeitos mais comuns de acontecer em idosos. Todas as alterações fisiológicas e cognitivas no idoso influenciam na segurança e efetividade do tratamento. Outro fator importante é a alta taxa de comorbidades clinicas e um potencial risco de interações medicamentosas que causam constipação, retenção urinária, sedação e hipotensão ortostática. Os benzodiazepínicos também estão associados ao número elevado de quedas e fraturas (CORDIOLI, 2011).

\section{Demência e uso de benzodiazepínicos}

A farmacoepidemiologia tem por seu atual desafio avaliar os efeitos de exposição à fármacos comuns sobre as doenças. Os distúrbios em relação as demências são um deles. Pois sabemos que sua incidência e prevalência são muito altas e aumentarão exponencialmente nas próximas décadas. Nenhum tratamento mostrou qualquer eficácia significativa, identificar e trabalhar com fatores de risco alteráveis, parece ser o caminho a seguir. Os distúrbios da demência ocorrem em idades em que as pessoas mais fazem o uso de drogas, correspondendo ao maior 
consumo de drogas durante o período da vida. Entretanto as drogas usadas excessivamente em idosos os benzodiazepínicos têm despertado o interesse dos pesquisadores. Seu uso é elevado entre os idosos, superior a 10\%. Na maioria dos países desenvolvidos são conhecidos pelos seus efeitos prejudiciais na memória e cognição. Embora, sejam boas ferramentas para o manejo à curto prazo de várias condições, como ansiedade e distúrbios do sono, elas tendem a ser mais prescritas a longo prazo. Por questões éticas óbvias, a ligação benzodiazepínicos-demência nos seres humanos são apenas observacionais. Os resultados podem ser alterados por fatores de confusão ou outros preconceitos com a causalidade reversa (quando prodromes da doença causam a exposição, ao invés da exposição ser potencialmente causadora da doença). Neste caso o controle adequado é complicado, uma vez que depressão e distúrbios do sono são frequentemente observados durante a fase prodrômica dos distúrbios da demência, e razões estas para prescrição de benzodiazepínicos. Uma associação positiva pode ser explicada pelo fato de que a exposição foi motivada pelos primeiros sintomas da doença (VERDOUX et al., 2005; COELHO et al., 2006; BILLIOTI DE GAGE et al., 2015).

Onze estudos foram publicados entre os anos de 1998 e 2015, sobre o uso dos benzodiazepínicos e o risco de distúrbios demenciais. Os vieses principais que poderiam explicar esses resultados contribuíram para uma confusão residual. Poderse-ia supor que a insônia é em si um fator de risco para a demência, que as pessoas com baixa reserva cognitiva são mais propensas a usar benzodiazepínicos, ou que seu estilo de vida está associado a menos interações sociais do que os não usuários, o que poderia contribuir para a construção e a associação estatística encontrada na ausência de qualquer relação causal. No entanto a maioria dos estudos tentou controlar esses viesses, considerando a existência de distúrbios de sono antes do tratamento. Como acontece frequentemente em farmacologia. Dos onze estudos publicados o tópico não era congruente: um concluiu que havia um efeito protetor, nove desses onze estudos encontraram um aumento nos transtornos de demência em usuários de benzodiazepínicos e apenas um deles não encontrou nenhuma associação. Apesar disso o conjunto de evidências parece justificar a prescrição e a renovação dos benzodiazepínicos somente se necessário. As únicas certezas do efeito dos benzodiazepínicos e a demência, são a ausência do efeito 
positivo nas placas de peptídeo-b amiloide e a sua ausência no desenvolvimento e propagação de emaranhados neurofibrilares. Os benzodiazepínicos podem ter um efeito causal precipitado em indivíduos com demência que poderiam ter vivido mais tempo sem sintomas, se não tivessem utilizado estas drogas (BILLIOTI DE GAGE et al 2012).

A maioria dos estudos farmacoepidemiológicos conduzidos até agora tem concluído que há um risco maior de distúrbios de demência em usuários de benzodiazepínicos, nenhum encontrou uma relação causal. Dá mesma forma nenhum desses estudos foi capaz de excluir a associação e de explica-la, unicamente pela presença de vieses. Tais estudos apresentam diferenças não só em resultados e conclusões, mas também em seus pontos fortes e limitações. Décadas separam o aparecimento dos fatores de risco/ansiedade e depressão das lesões cerebrais iniciais e o começo da demência e seus distúrbios associados até o seu diagnóstico. É necessário para o estudo adequado com benzodiazepínicos, do ponto de vista da saúde pública seria um tempo de trinta anos com acompanhamento contínuo e avaliação de fatores de risco como depressão, distúrbio do sono e ansiedade. É sabido que nenhum estudo farmacoepidemiologico consiga combinar todos esses requisitos, o que deixa margem para dúvidas quanto a natureza da associação. Permanece incerto a fisiopatologia da demência e os efeitos dos benzodiazepínicos nos estágios de desenvolvimento da doença, para se ter qualquer certeza provavelmente não existe nenhum argumento a favor do risco aumentado de demência associado a uma utilização racional de benzodiazepínicos, desde que seu uso não ultrapasse três meses. O conjunto de provas relativas ao uso prolongado de benzodiazepínicos cujo benefício não foi demonstrado é importante e coerente para ser ignorado, porque o nível de conhecimento experimental e observacional é insuficiente para excluir a possibilidade de uma associação causal. É de extrema importância aumentar os esforços de pesquisa e apoio sobre essa questão, somente assim possibilitaram que uma hipótese causal seja descartada, garantindo aos pacientes, profissionais e autoridades a respeito dessa preocupação (BILLIOTI DE GAGE et al 2012).

$\mathrm{O}$ uso de benzodiazepínicos em idosos pode comprometer e desenvolver deficiências cognitivas que persistem durante meses após a suspensão do uso, os 
idosos são mais suscetíveis aos seus efeitos. Longevos com comprometimento cognitivo devem ser investigados quanto ao uso crônico de benzodiazepínicos (BOTTINO et al., 2011).

Estudos experimentais e clínicos documentaram efeitos agudos dos benzodiazepínicos sobre o desempenho da atenção e memória, os efeitos do uso prolongado dos mesmos no funcionamento cognitivo são mais difíceis de avaliar. Estudos do tipo transversal e de casos- controle que investigaram esses efeitos comparando usuários crônicos de benzodiazepínicos com usuários não crônicos, levantam questões metodológicas limitando suas conclusões. A maioria dos estudos clínicos longitudinais dos pacientes que tomam benzodiazepínicos tem uma pequena amostra e tamanho, um seguimento curto e nenhum grupo controle apropriado. Muitas questões sobre o comprometimento cognitivo do seu uso prolongado estão em aberto. A natureza e a gravidade do comprometimento cognitivo dos usuários crônicos, o possível desenvolvimento da tolerância, e a reversibilidade dos distúrbios cognitivos, mas seus efeitos negativos necessitam de uma investigação mais aprofundada. De acordo com as recomendações do comitê de E Deug Administratiom 4 e o EU National Health and 5, o uso de benzodiazepínicos não deve exceder a quatro meses. Porém a prevalência do uso de benzodiazepínicos nos Estados Unidos diminuiu ao longo do período de 1978 a 1987, mas grande número de pacientes ainda são tratados por períodos de sete a dez meses. O uso prolongado de benzodiazepínicos pode acelerar o declínio cognitivo em idosos, poucos estudos populacionais examinaram a associação entre o declínio cognitivo e o uso de benzodiazepínicos, porém o presente estudo fez a avaliação num período de quatro anos e associou o risco aumentado de declínio cognitivo em longevos (PATERNITI e ALPÉROVITCH, 2002).

Um estudo de corte em que os indivíduos foram observados 5 vezes em 22 anos mostrou que de 1134 homens, 103 (9,1\%) administraram benzodiazepínicos regularmente em uma ou mais fases. A administração regular de benzodiazepínicos mostrou ser um fator de risco para a demência nestes homens (GALLACHER et al., 2012).

Uma meta-análise conduzida por ZHONG et al. (2015) mostrou que o uso de benzodiazepínicos por um longo tempo pode aumentar o risco para desenvolver 
demência. Além disso, um estudo que utilizou diferentes metodologias, algoritmos e banco de dados mostrou que o uso prolongado de benzodiazepínicos apresenta forte associação com o risco aumentado em desencadear demências (TAKADA et al., 2016).

Um recente estudo caso-controle com 91 indivíduos caso e 182 indivíduos controles com idade igual ou superior a 75 anos, observou que indivíduos chineses podem desenvolver demência quando administram altas doses de benzodiazepínicos (CHAN et al., 2017).

Vários estudos evidenciaram um risco aumentado de demência em usuários de benzodiazepínicos. A incidência de demência e o uso de benzodiazepínicos são altos, particularmente nos idosos. Portanto, mesmo que se tenha um aumento moderado desse risco, isso poderá induzir uma enorme quantidade de casos e terá um grande impacto na saúde pública (Zhong et al., 2015; BILLIOTI DE GAGE et al., 2015; Chan et al., 2017).

\section{CONCLUSÃO}

Segundo estudos o uso prolongado de benzodiazepínicos em idosos pode ter um risco moderado para o desenvolvimento da demência. Porém, sabe-se que vários sintomas que antecedem a demência na idade avançada são tratados com esses fármacos, como a insônia e a ansiedade. Há indicativo de que o uso de benzodiazepínicos induz comprometimento cognitivo e aumenta o número de quedas destes indivíduos. O uso prolongado leva à uma série de prejuízos principalmente em idosos, por isso todo o cuidado tem que ser tomado na hora da escolha do uso e tempo de tratamento por conta dos efeitos colaterais que são mais intensificados em idosos. O número de pessoas que irão desenvolver a demência cresce a cada dia, por isso sabe-se com certeza é que existe uma necessidade global de mais estudos e pesquisas em relação essa doença e a utilização desses fármacos. Estudos apontam um risco aumento para os indivíduos idosos em desenvolver demências com o uso prolongado de benzodiazepínicos. A população mundial está envelhecendo e, portanto, precisa-se do desenvolvimento de políticas públicas de saúde para as pessoas com mais de 60 anos para que estas sejam 
assistidas e que, inclusive, se possa ter maiores informações acerca do efeito prolongado de certos medicamentos com os benzodiazepínicos. Adicionais pesquisas relacionadas aos fármacos que possam ser utilizados por idosos e que não comprometam a saúde dos mesmos e sim aumentam a qualidade de vida destes, são necessários.

\section{REFERÊNCIAS}

Bernik, Márcio. Aspectos clínicos e farmacológicos dos tranquilizantes benzodiazepínicos: 1. ed. São Paulo: Edimédica, 2010.

Billioti de Gage S, Bégaud B, Bazin F, Verdoux H, Dartigues JF, Pérès K, Kurth T, Pariente A. Benzodiazepine use and risk of dementia: prospective population based study. Bmj, 2012; 345: e6231.

Billioti de Gage S, Pariente A, Bégaud B. Is there really a link between benzodiazepine use and the risk of dementia? Expert Opin Drug Saf. 2015; 14(5):733-47.

Bottino, Cássio MC et al. Diagnóstico diferencial entre demência e transtornos psiquiátricos. 2011.

Castro, Gustavo Loiola Gomes et al. Uso de Benzodiazepínicos como automedicação: consequências do uso abusivo, dependência, farmacovigilância e farmacoepidemiologia. Revista Interdisciplinar, v. 6, n. 1, p. 112-123, 2013.

Chan TT, Leung WC, Li V, Wong KW, Chu WM, Leung KC, Ng YZ, Kai YG, Shea YF, Chang SR, Chu LW. Association between high cumulative dose of benzodiazepine in Chinese patients and risk of dementia: a preliminary retrospective case-control study. Psychogeriatrics. 2017.

Coelho, Fernando Morgadinho Santos et al. Benzodiazepínicos: uso clínico e perspectivas. Rev Bras Med, 2006; 63 (5): 196-200.

Cordioli,Aristides Volpato et al.Psicofármacos: consulta rápida.4.ed.Porto Alegre:

Custodio N, Wheelock A, Thumala D, Slachevsky A. Dementia in Latin America: Epidemiological Evidence and Implications for Public Policy. Front Aging Neurosci. 2017; 13;9:221.

Cruz, A. V. et al. Uso crônico de diazepam em idosos atendidos na rede pública em Tatuí-SP. Rev Ciênc Farm Básica Apl, 2006; 27(3): 259-67. 
Faustino, Christine Grützmann; MARTINS, M. A.; JACOB-FILHO, Wilson. Medicamentos potencialmente inapropriados prescritos a pacientes idosos ambulatoriais de clínica médica. Einstein (Sao Paulo), 2011; 9(1): 18-23.

Forsan, Maria Aparecida. O USO INDISCRIMINADO DE BENZODIAZEPÍNICOS: UMA ANÁLISE CRÍTICA DAS PRÁTICAS DE PRESCRIÇÃO, DISPENSAÇÃO E USO PROLONGADO. Campos Gerais: Universidade Federal de Minas Gerais, 2010; 11.

François M, Sicsic J, Elbaz A, Pelletier Fleury N. Trends in Drug Prescription Rates for Dementia: An Observational Population-Based Study in France, 2006-2014. Drugs Aging. 2017.

Gallacher J, Elwood P, Pickering J, Bayer A, Fish M, Ben-Shlomo Y. Benzodiazepine use and risk of dementia: evidence from the Caerphilly Prospective Study (CaPS). J Epidemiol Community Health. 2012; 66(10):869-73.

Garrido, Regiane; MENEZES, Paulo R. O Brasil está envelhecendo: boas e más notícias por uma perspectiva epidemiológica. Rev bras psiquiatr, 2002; 24(1): 3-6.

Ismail Z, Nguyen MQ, Fischer CE, Schweizer TA, Mulsant BH, Mamo D. Neurobiology of delusions in Alzheimer's disease. Curr Psychiatry Rep. 2011;13(3):211-8.

Linden M, Bär T, Helmchen H: Prevalence and appropriateness of psychotropic drug use in old age: results from the Berlin Aging Study (BASE). Int Psychogeriatr 2004;16:461-480.

Oliveira, Adriana B. Pillegi et al. Clínica psiquiátrica. Barueri, SP.2011.

ONU, United Nations. World Population Ageing 2013 [Internet]. New York, USA: Department of Economic and Social Affairs, Population Division; 2013. Report No.: ST/ESA/SER.A/348. Available: http://www. un.org/en/development/desa/population/ publications/pdf/ageing/WorldPopulationAgeing2013.pdf.

ONU, United Nations, Department of Economic and Social Affairs, Population Division (2015). World Population Ageing 2015 (ST/ESA/SER.A/390).

PATERNITI, Sabrina; DUFOUIL, Carole; ALPÉROVITCH, Annick. Long-term benzodiazepine use and cognitive decline in the elderly: the Epidemiology of Vascular Aging Study. Journal of clinical psychopharmacology, v. 22, n. 3, p. 285293, 2002.

Pincus HA, Pike KM, Spaeth-Rublee B, Elinson L. Health and Aging Policy Fellows Program: Shaping a Healthy Future for Older Americans. J Am Geriatr Soc. 2017. 


\section{Artigo Original}

\section{Fisiopatologia}

Ramos, Luiz Roberto. Fatores determinantes do envelhecimento saudável em idosos residentes em centro urbano: Projeto Epidoso, São Paulo Determinant factors for healthy aging among senior citizens in a large city: the Epidoso. Cad. saúde pública, 2003; 19 (3): 793-798.

Sanderson WC, Scherbov S. Faster increases in human life expectancy could lead to slower population aging. PLoS One. 2015; 15;10(4):e0121922.

Takada M, Fujimoto M, Hosomi K. Association between Benzodiazepine Use and Dementia: Data Mining of Different Medical Databases. Int J Med Sci. 2016; 13(11):825-834.

Tazkarji B, Lam R, Lee S, Meiyappan S. Approach to preventive care in the elderly. Can Fam Physician. 2016 Sep;62(9):717-21.

Vaz, Célia Sofia Silva Bidarra. Medicamentos potencialmente inapropriados em idosos: a realidade de um Serviço de Medicina. 2012. Dissertação de Mestrado.

Verdoux $\mathrm{H}$, Lagnaoui $\mathrm{R}$, Begaud $\mathrm{B}$. Is benzodiazepine use a risk factor for cognitive decline and dementia? A literature review of epidemiological studies. Psychol Med. 2005 Mar;35(3):307-15.

WHO. World Health Organization. Envelhecimento ativo: uma política de saúde / World Health Organization; tradução Suzana Gontijo. - Brasília: Organização PanAmericana da Saúde, 2005. 60p.: il.

Zhong G, Wang Y, Zhang Y, Zhao Y. Association between Benzodiazepine Use and Dementia: A Meta-Analysis. PLoS One. 2015; 27;10(5):e0127836. 